\title{
DEFORMATION TWINNING AND ITS EFFECT ON CRACK EXTENSION
}

\author{
Q. H. TANG $\dagger$ and T. C. WANG
}

LNM Institute of Mechanics, Chinese Academy of Sciences, Beijing 100080, China

(Received 6 February 1998; accepted 4 June 1998)

\begin{abstract}
Deformation twinning near a crack tip is observed in b.c.c. metal Mo based on molecular dynamics simulation at temperature $T=50 \mathrm{~K}$ and loading rate $\dot{K}_{\mathrm{II}}=0.0706 \mathrm{MPa} \mathrm{m}{ }^{1 / 2} / \mathrm{ps}$. The deformation twinning is closely controlled by both the crystal geometry orientation and the stress distribution. The width of the deformation twin band is affected by the distance between the upper and lower crack surfaces. The twin plane and twin direction are $(1 \overline{1} 2)$ and [ $\overline{1} 11]$, respectively. The initial crack extension occurs in the deformation twin region near the crack tip. The simulation shows that the extension direction of the crack is changed as the crack propagates over the twinning boundary. (C) 1998 Acta Metallurgica Inc. Published by Elsevier Science Ltd. All rights reserved.
\end{abstract}

\section{INTRODUCTION}

Most textbooks on strength and ductility of materials pay more attention to the slip processes and associated dislocation movement and pay less attention to the deformation twinning.

In recent years, it has been known that deformation twinning strongly influences the strength and ductility of some interesting materials $[1,2]$, which has attracted more researchers to study deformation twinning. Morris et al. [3] studied the structure and energies of compression twin boundaries using the embedded-atom techniques. Serra and Bacon [4] simulated the atomic structure, energy and the stress state of the planar twin interface by using a many body potential. Yoo and Lee [5] studied the twin nucleation mechanisms and the anisotropic coupling effect of normal stresses on twin dislocation mobility. But at present, there is little understanding of what factors determine whether or not a material will easily form twins. Some experiments on silicon deformation at low temperature $[6,7]$ indicated that with decreasing temperature, twinning becomes more important as a mode of deformation, whereas at temperatures

$\dagger$ To whom all correspondence should be addressed. above the brittle-ductile transition of silicon, the primary mode of deformation occurs by the glide of slip dislocation. Schlogl and Fischer [8] studied the anisotropic plastic behavior of a single grain with a lamellar microstructure. In their work, the main deformation mechanisms of ordinary slip and deformation twinning were considered.

In this paper, for the b.c.c. metal Mo, both dislocation emission and deformation twinning are studied for the different orientation samples under the fixed displacement boundary. The pure shear load and the mixed mode loads are discussed in detail.

\section{CALCULATION METHOD}

\subsection{The interatomic potential}

The " $N$-body" potential $M_{0}$ proposed by Tang $e t$ al. [9] is used here, the total energy $E_{\mathrm{T}}$ of an assembly of atoms is

$$
E_{\mathrm{T}}=\frac{1}{2} \sum_{i, j, i \neq j} \phi\left(r_{i j}\right)+\sum_{i} F\left(\frac{\rho_{i}}{\rho_{\mathrm{e}}}\right)
$$

where $r_{i j}$ is the distance between atoms $i$ and $j$, $\phi\left(r_{i j}\right)$ the pair-potential, and $F\left(\rho_{i} / \rho_{\mathrm{e}}\right)$ the embedding energy function. They are described by the following formulas:

$$
\begin{aligned}
\phi\left(\frac{r_{i j}}{r_{1 \mathrm{e}}}\right)= & B_{0}+B_{1}\left(\frac{r_{i j}}{r_{1 \mathrm{e}}}-1\right)+B_{2}\left(\frac{r_{i j}}{r_{1 \mathrm{e}}}-1\right)^{2}+B_{3}\left(\frac{r_{i j}}{r_{1 \mathrm{e}}}-1\right)^{3}+H\left(r_{i j}-r_{2 \mathrm{e}}\right)\left[B_{4}\left(\frac{r_{i j}}{r_{1 \mathrm{e}}}-1\right)^{3}\right. \\
& \left.+B_{5}\left(\frac{r_{i j}}{r_{1 \mathrm{e}}}-1\right)^{3}\left(\frac{r_{\mathrm{c}}}{r_{2 \mathrm{e}}}-1\right)\right]\left(\frac{r_{\mathrm{c}}}{r_{2 \mathrm{e}}}-1\right)^{3}
\end{aligned}
$$




$$
F^{\prime}\left(\frac{\rho}{\rho_{\mathrm{e}}}\right)= \begin{cases}-10 \sqrt{0.0515-\left(\frac{\rho}{\rho_{\mathrm{e}}}-0.778\right)^{2}}+1.9, & 0.68 \leq \frac{\rho}{\rho_{\mathrm{e}}} \leq 0.775 \\ -0.72\left(\frac{\rho}{\rho_{\mathrm{e}}}-0.775\right)-0.3, & 0.775 \leq \frac{\rho}{\rho_{\mathrm{e}}} \leq 0.83 \\ -10 \sqrt{0.397-\left(\frac{\rho}{\rho_{\mathrm{e}}}-0.88\right)^{2}}+0.59, & 0.83 \leq \frac{\rho}{\rho_{\mathrm{e}}} \leq 1.01\end{cases}
$$

where $\rho$ and $\rho_{\mathrm{e}}$ are the atomic electron density in the deformed and undeformed states, respectively. $r_{1 \mathrm{e}}$ and $r_{2 \mathrm{e}}$ are the first- and second- neighbor atom distances, and $r_{\mathrm{c}}$ is the cutoff distance of $N$-body potential. The coefficients $B_{i}(i=1, \ldots, 6)$ in equation (2) were given in Ref. [9]. $H\left(r_{i j}-r_{2 \mathrm{e}}\right)$ is the step function.

\subsection{Atom lattice geometry}

2.2.1. Sample 1. The parallelepiped with a slit is used as the simulated model in which the slip plane is coincident with the crack plane. The coordinate system is selected to be the $x-, y$-, and $z$-axes along

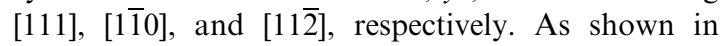
Fig. 1(a) and (b), the size of the atomic area is $500 \times(\sqrt{3} / 6) a_{0}$ in the $x$-direction, $40 \times(\sqrt{2} / 2) a_{0}$ in the $y$-direction, and $6 \times(\sqrt{6} / 6) a_{0}$ in the $z$-direction, and $a_{0}$ is a lattice constant. Along the $x$-direction,

(a)

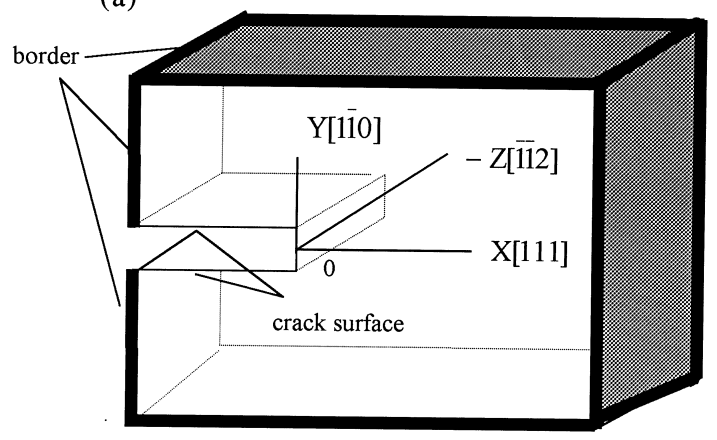

(b) $\mathrm{Y}[1 \overline{1} 0]$ the periodicity is in three layers of (111) atomic planes and its length is $3 \times(\sqrt{3} / 6) a_{0}$; along the $y$ direction, the periodicity is in two layers of (110) atomic planes and its length is $2 \times(\sqrt{2} / 2) a_{0}$; along the $z$-direction, the periodicity is in six layers of $(11 \overline{2})$ atomic planes and the length is $6 \times\left(\frac{\sqrt{6}}{6}\right) a_{0}$. The left-hand side of the boundary to the crack tip is $120 \times(\sqrt{3} / 6) a_{0}$; the separation of the upper and lower crack surface is taken to be $\sqrt{2} a_{0}$ which is of two layers of atomic planes (a periodicity) along [1]̄0]; the number of atoms in the present simulation is about 20000 .

2.2.2. Sample 2. The coordinate system is selected to be $x$-, $y$-, and $z$-axes along [111], [112], and [110], respectively, as shown in Figs 2(a) and (b). The separation distance of the upper and lower crack surfaces is taken to be $2.4 a_{0}$ which is of six layers of atomic planes (a periodicity) along [112]. Along $x$ (a)

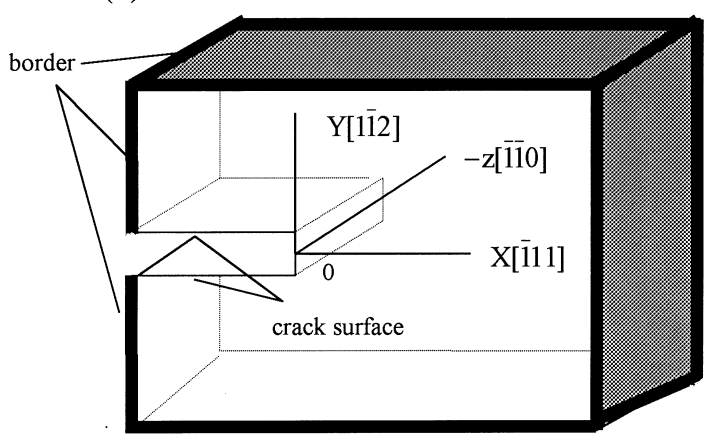

(b)

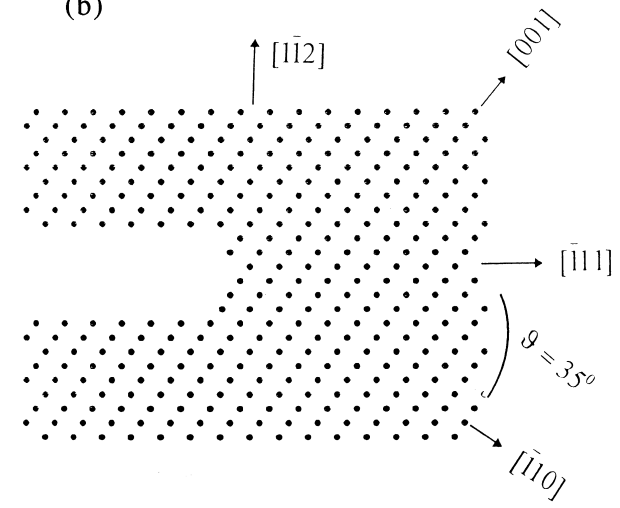

Fig. 1. (a) Schematic diagram of the simulated sample. (b) Atomic configuration near the crack tip.

Fig. 2. (a) Schematic diagram of the simulated sample. (b) Atomic configuration near the crack tip. 
[111], the length is $440 \times(\sqrt{3} / 6) a_{0}$, along $y$ [11 2 , the length is $120 \times(\sqrt{6} / 6) a_{0}$, and along $z$ [110], the length is $2 \times(\sqrt{2} / 2) a_{0}$. The number of atoms in the present simulation is about 20000 .

2.2.3. Sample 3. The coordinate system is selected to be $x-, y$-, and $z$-axes along [110], [001], and [110], respectively. Compared to sample 2, the $z$-axis is still along [110]. The axes $x, y$ rotate $\theta=35^{\circ}$ around the $z$-axis.

\subsection{The boundary condition}

The fixed displacement boundary scheme $[10,11]$ is adopted in this paper. A prescribed displacement distribution dictated by the linear elastic $\mathrm{K}$ field in the $x-y$ plane is applied to the atoms on the border of the inner discrete system, and along the $z$-direction, a six layer periodic representation is applied. In the following simulation, the loading scheme is with the pure mode II singularity field or with the mixed mode I and mode II loads singularity field of the plane strain, respectively.

The crack tip geometry is constructed by removing two layers of (11̄0) atomic planes which is just one periodicity along the $y$-direction in sample 1 , see Figs 1(a) and (b), and six layers of (11/2) atomic planes which are just one periodicity along the $y$ direction in sample 2, see Figs 2(a) and (b). Because the opening distance $\sqrt{2} a_{0}$ of crack faces in sample 1 or $2.4 a_{0}$ in sample 2 is greater than the cutoff distance $r_{\mathrm{c}}$ of " $N$-body" potential which is being used, $r_{\mathrm{c}}=1.25 a_{0}$. The advantage is that atoms in the upper and below atomic planes do not interact with each other naturally.

The results obtained by Gumbsch [12] and Paskin et al. [13] show that slightly blunted crack tips propagated at loads which were only marginally higher than the load for brittle crack propagation of a sharp crack. The reason for this result is attributed to the nonlinearity of atomic interaction. In our simulation, we use a slight blunted crack tip instead of a sharp crack. Based on the studies by Gumbsch and Paskin et al. $[12,13]$, we can presume that such a choice of crack tip geometry only has a marginal effect on the simulation results.

\subsection{The calculation method}

Inner atoms of samples follow Newton's law

$$
F_{i}=-\frac{\partial E_{\mathrm{T}}}{\partial r_{i}}=m_{i} \dot{v}_{i}
$$

The present molecular dynamics calculations are carried out by the Leapfrog Algorithm [14] as follows:

$$
\left\{\begin{array}{l}
v_{i}\left(t+\frac{\Delta t}{2}\right)=v_{i}\left(t-\frac{\Delta t}{2}\right)+\frac{F_{i}}{m_{i}} \Delta t \\
r_{i}(t+\Delta t)=r_{i}(t)+v_{i}\left(t+\frac{\Delta t}{2}\right) \Delta t \\
v_{i}(t+\Delta t)=\frac{1}{2}\left[v_{i}\left(t+\frac{\Delta t}{2}\right)+v_{i}\left(t-\frac{\Delta t}{2}\right)\right]
\end{array}\right.
$$

where $m_{i}$ and $v_{i}$ are the mass and velocity of the $i$ th atom, respectively, $F_{i}$ the force acting on the $i$ th atom, and $r_{i}$ the distance of atom $i$ with respect to the origin of the coordinate system.

The loading rate is $\dot{K}_{\mathrm{II}}=0.0706 \mathrm{MPa} \mathrm{m}^{1 / 2} / \mathrm{ps}$, the stress intensity factor $K_{\mathrm{II}}$ is chosen as the loading parameter. The time step $\Delta t$ is $1.256 \times 10^{-14} \mathrm{~s}$.

\subsection{The temperature condition}

The initial velocities of atoms are the Maxwellian distribution corresponding to a given temperature. It is assumed that the simulated system is connected to a large heat reservoir with a certain temperature which provides enough heat energy to maintain the system at the fixed value throughout the duration of the runs. This means that any small disturbance in temperature due to loading or the dislocation emission can be neglected $[14,15]$.
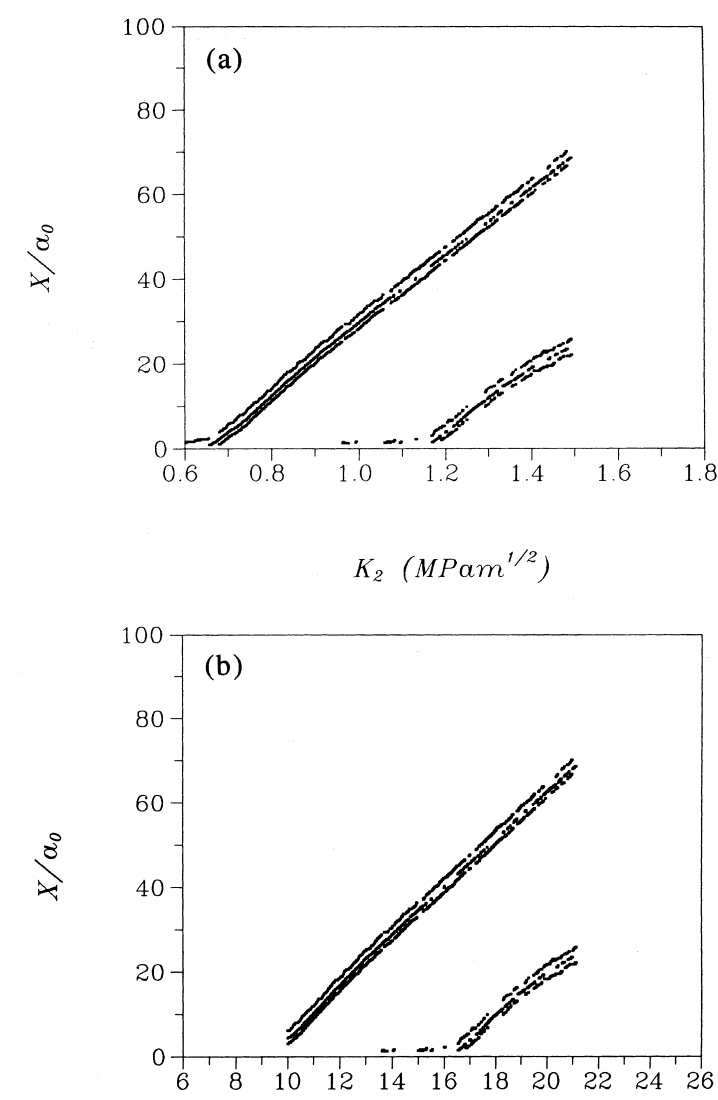

Fig. 3. Dislocation emission: (a) emitted dislocation position vs stress intensity factor; (b) emitted dislocation position vs time. 


\section{RESULTS AND DISCUSSION}

\subsection{Case 1}

Sample 1 is simulated under pure mode II load. The emission of dislocations has been observed near the crack tip. The normal direction of the slipping plane and slip direction are [110] and [111], respectively. The positions of the two full emitted dislocations vs the stress intensity factor are plotted in Fig. 3(a). As $K_{\mathrm{II}}$ reaches about $0.668 \mathrm{MPa} \mathrm{m}^{1 / 2}$, the first full dislocation has been emitted. As $K_{\mathrm{II}}$ reaches about $1.17 \mathrm{MPa} \mathrm{m}^{1 / 2}$, the second full dislocation has been observed. The dislocation position vs time is plotted in Fig. 3(b) from which the dislocation velocity can be evaluated, about 1800 $1950 \mathrm{~m} / \mathrm{s}$. A full dislocation is always dissociated into three partial dislocations for b.c.c. metal Mo in the present simulation. The atom configuration near the crack tip is shown in Figs 4(a) and (b) from which the two full dislocations (six partial dislocations) can be observed clearly.

\subsection{Case 2}

Sample 2 is simulated under pure mode II load. Compared to case 1, except for the geometry orientation of the sample, other conditions such as temperature, loading rate, and the fixed displacement boundary scheme are the same. The deformation twinning occurs at $K_{\mathrm{II}}=0.975 \mathrm{MPa} \mathrm{m}^{1 / 2} \quad$ [see Fig. 5(a)]. The twin plane and twin direction are (112) and [111], respectively, which is in good agreement with experimental observation [16]. The width of the deformation twin band is the same as that of the opening distance of the upper and lower crack surfaces. And the deformation twin band has been extended to a distance of $12 a_{0}$ from the crack tip. We can conclude that the deformation twinning nucleates initially from the crack tip where there is

(a)

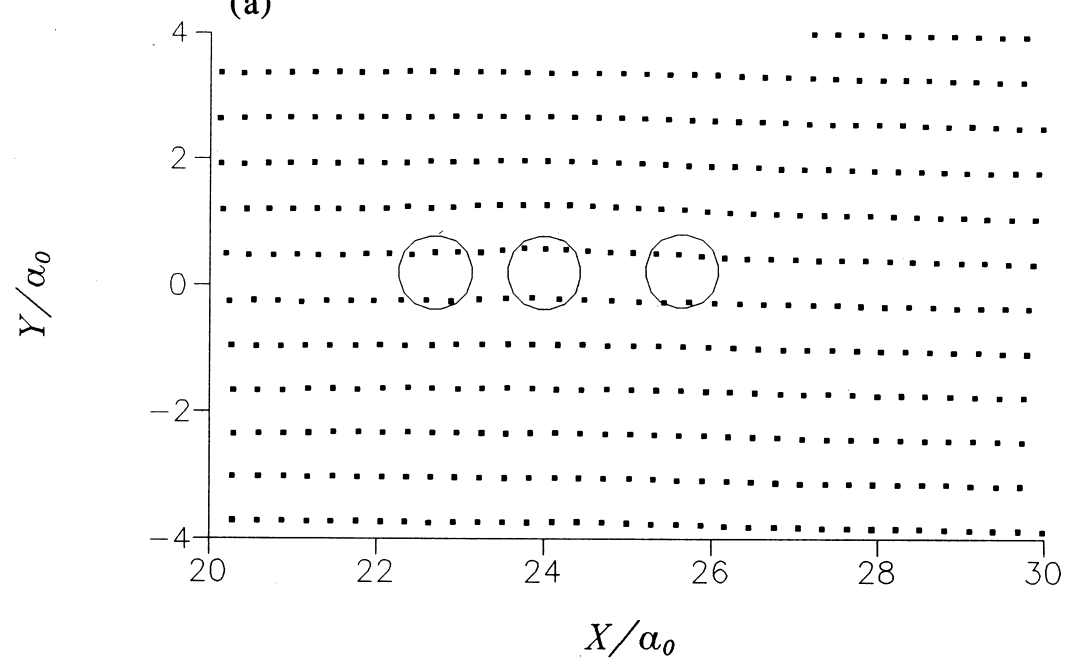

(b)

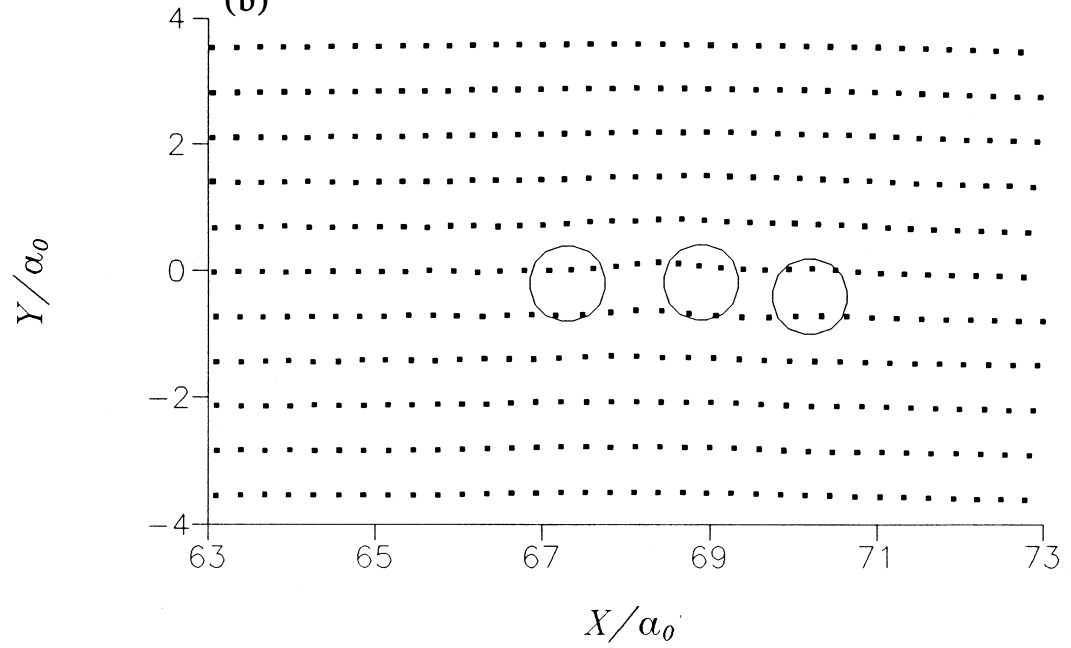

Fig. 4. Dislocation emission: (a), (b) atomic configuration near the crack tip, $K_{\mathrm{II}}=1.17 \mathrm{MPa} \mathrm{m}{ }^{1 / 2}$. 
TANG and WANG: DEFORMATION TWINNING
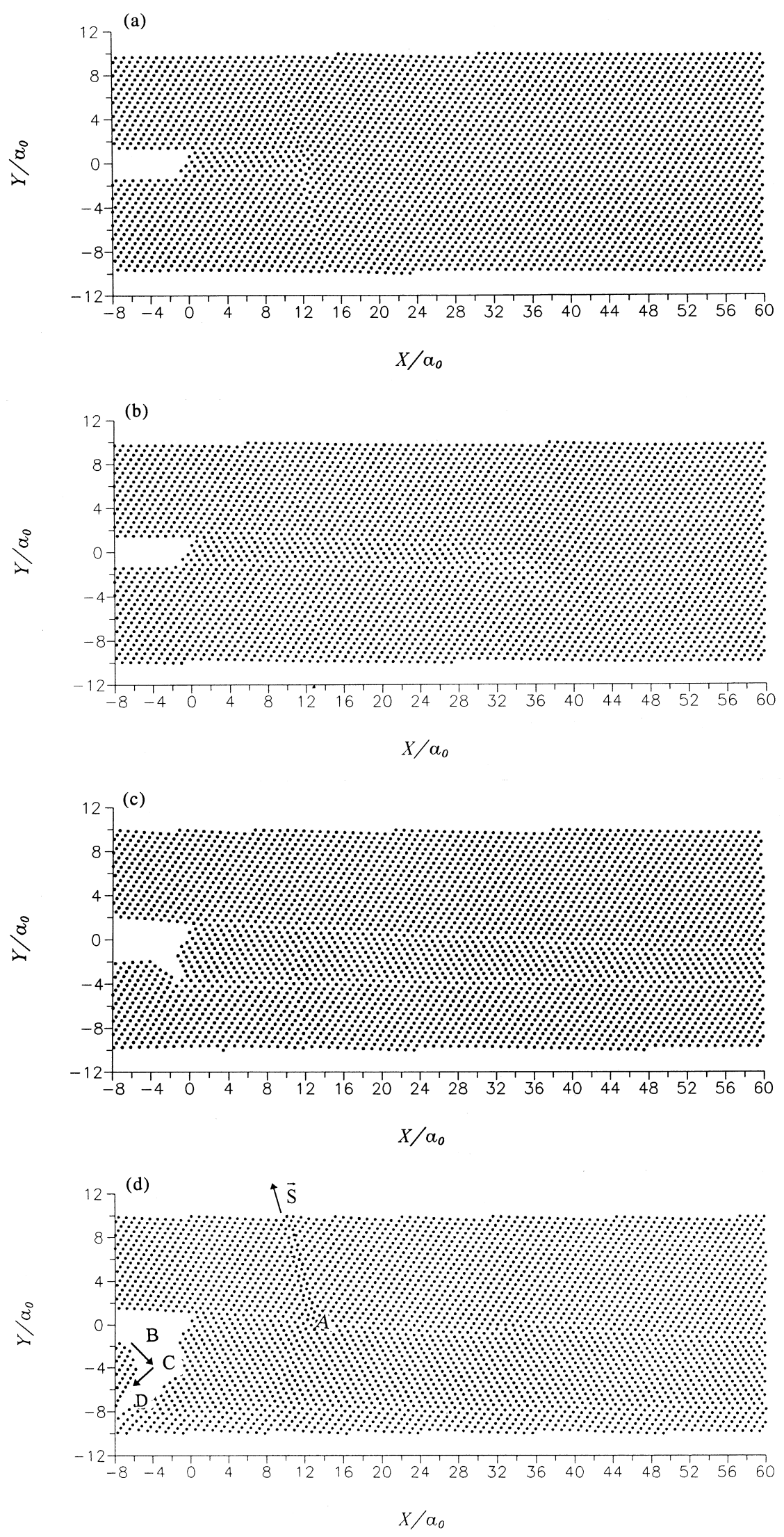

Fig. 5. Atomic configuration near the crack tip, deformation twinning band and crack extension under pure shear load for sample 2: (a) $K_{\mathrm{II}}=0.975 \mathrm{MPa} \mathrm{m}^{1 / 2}$; (b) $K_{\mathrm{II}}=1.240 \mathrm{MPa} \mathrm{m}^{1 / 2}$; (c) $K_{\mathrm{II}}=3.725 \mathrm{MPa} \mathrm{m}^{1 / 2} ;$ (d) $K_{\mathrm{II}}=4.612 \mathrm{MPa} \mathrm{m}^{1 / 2}$. 
high stress concentration. As $K_{\mathrm{II}}=1.24 \mathrm{MPa} \mathrm{m}^{1 / 2}$, the deformation twinning has extended to a distance of about $32 a_{0}-34 a_{0}$ from the crack tip. The extensive velocity of the deformation twinning can be derived from Figs $5(\mathrm{a})$ and (b) as about $850 \mathrm{~m} / \mathrm{s}$ which is less than half of the glide velocity of edge dislocations. The width of deformation twin band gradually becomes narrow along the $x$-axis. The reason is that the stress level reduces with increasing distance from the crack tip along the $x$-axis. So there is a close relation between the deformation twinning and stress distribution. As $K_{\mathrm{II}}=3.725 \mathrm{MPa} \mathrm{m}^{1 / 2}$, the crack propagation has been observed in Fig. 5(c). The initial propagation of a crack is in the twin interface. Several factors are responsible for this result. Firstly, there is high stress concentration and large deformation near the crack tip; secondly, the tension strength in the metal materials generally is less than the compressive strength, and atoms of the upper region of the crack plane are in the state of compression deformation and the lower region in the state of tensile deformation in the present simulation. Thirdly, after the deformation twinning, the crystal orientation within the deformation twin band is changed, and forms the stable structure which prevents the crack propagation along the crack plane. Therefore, it can be understood that the crack can propagate in the right and lower region of the crack plane [see Figs 5(c) and (d)].

The crack propagates continuously as the external load is increased. And it is very interesting that the deformation twin band becomes wider with the opening distance of the crack surfaces. The direction of crack propagation at position $\mathrm{C}$ is changed from originally along the direction of $\mathrm{BC}$ to along CD. The position $\mathrm{C}$ in Fig. 5(c) was in the twin interface from which the conclusion can be derived. As the propagating crack runs into the twin boundary, the propagating direction of the crack may be changed. As the external stress intensity factor

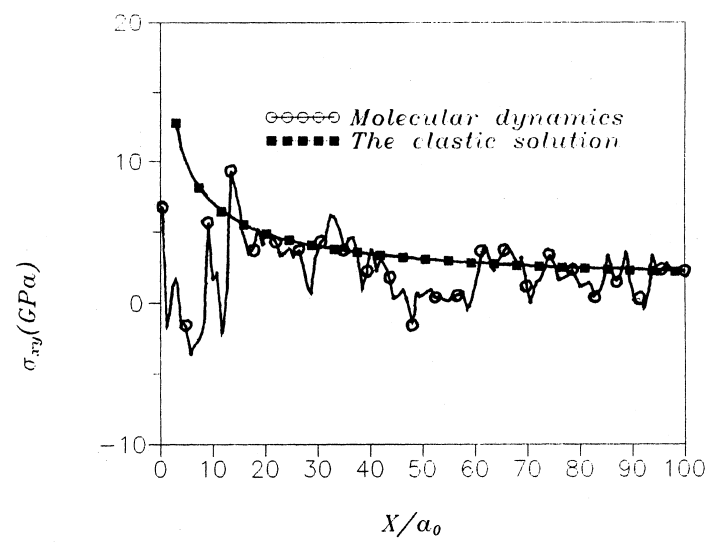

Fig. 6. Shear stress distribution along the $x$-axis under pure shear load for sample $2, K_{\mathrm{II}}=0.975 \mathrm{MPa} \mathrm{m}^{1 / 2}$.

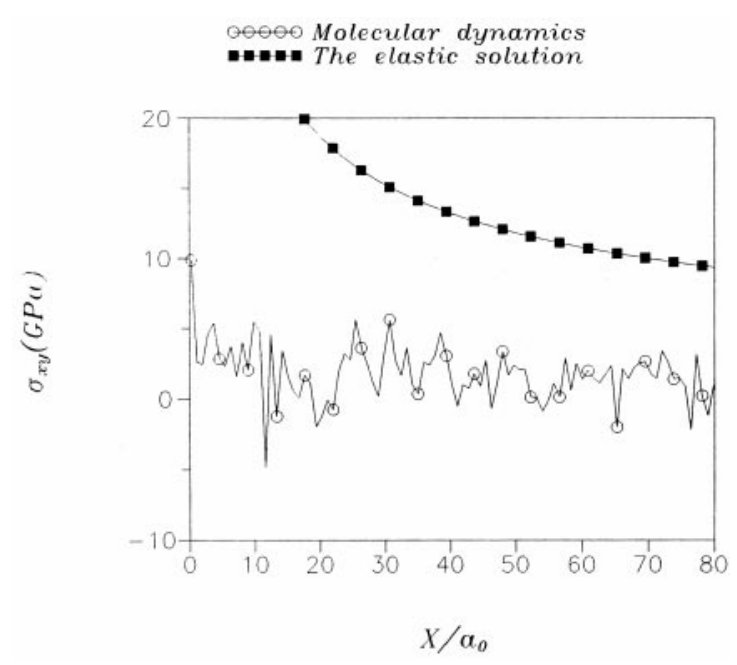

Fig. 7. Shear stress distribution along the $x$-axis under pure shear load for sample $2, K_{\mathrm{II}}=3.725 \mathrm{MPa} \mathrm{m}^{1 / 2}$.

reaches $4.612 \mathrm{MPa} \mathrm{m}^{1 / 2}$ which is $0.887 \mathrm{MPa} \mathrm{m}^{1 / 2}$ higher than that of the deformation state of Fig. 5(c), the propagating velocity of the crack is very slow which is contributed to by two factors. One is the change of crack propagating route; the other is that the deformation twin band becomes wider which certainly dissipates some energy.

A little mismatch of atoms at point $\mathrm{A}$ near the twin boundary at a distance of $12 a_{0}$ from the crack tip can be found in Fig. 5(d). This tiny defect leads to a little slip along the $S$-direction. Because the external load reaches $4.612 \mathrm{MPa} \mathrm{m}^{1 / 2}$ which is very high, any tiny defect in the region of high stress concentration may cause other defects.

The shear stress distribution along the $x$-axis is shown in Fig. 6 at a load level of $K_{\mathrm{II}}=0.975 \mathrm{MPa} \mathrm{m}^{1 / 2}$. Combining Fig. 5(a) with Fig. 6 , as $x / a_{0}>12$, the simulated result is in good agreement with the elastic solution; but as $x / a_{0}<12$, there is remarkable deviation from the elastic solution. It is reasonable that within a distance of $12 a_{0}$ from the crack tip, there is a deformation twin band which is not in the state of pure elastic deformation. Figure 7 also shows the shear stress distribution at a load level of $K_{\mathrm{II}}=3.725 \mathrm{MPa} \mathrm{m}^{1 / 2}$ at which the deformation twin band is extended beyond the distance $60 a_{0}$ from the crack tip along the $x$-direction. The stress distribution obtained in the MD method is nearly homogeneous, about 2$3 \mathrm{GPa}$, which is contributed by the homogeneous large shear deformation in the deformation twin band. The stress distribution obtained by the elastic solution is higher than that of MD, because the stress in the process of deformation twinning is relaxed [16].

The loading rate in the MD simulation is very high, the time step is in units of ps or fs. At the same loading rate, the dislocation emission can be 


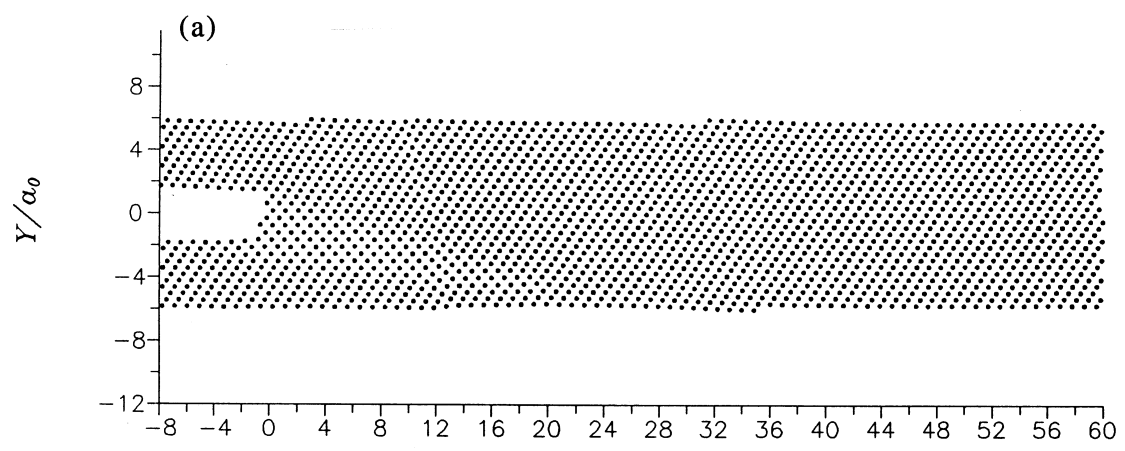

$X / a_{0}$

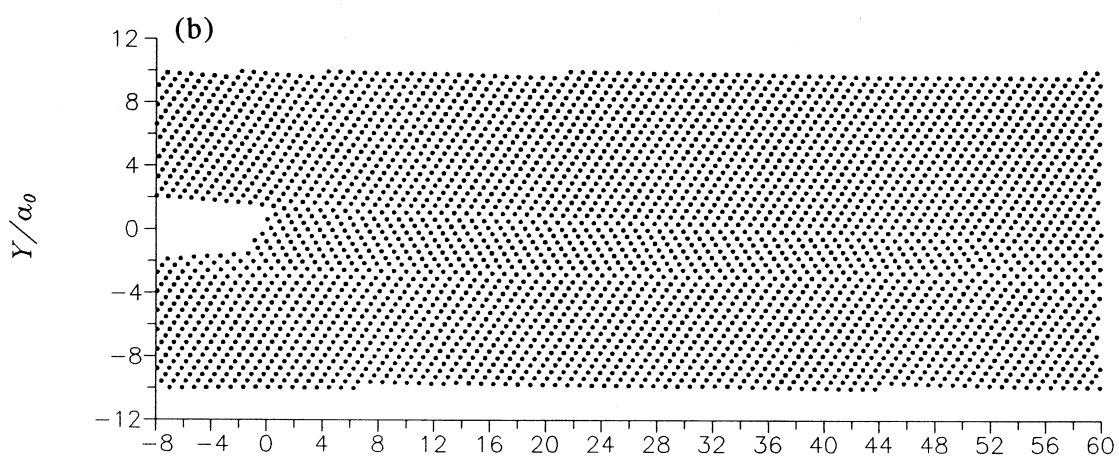

$X / a_{0}$

(c)

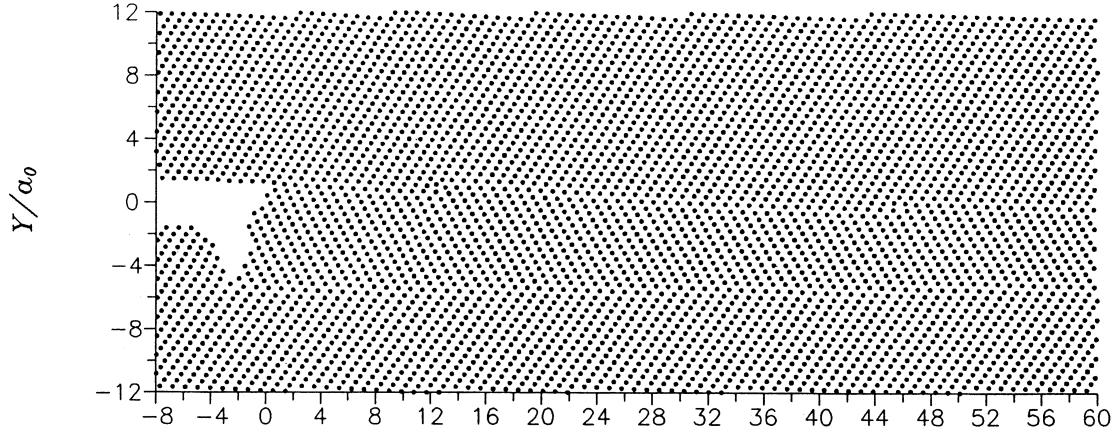

$X / a_{0}$

(d)

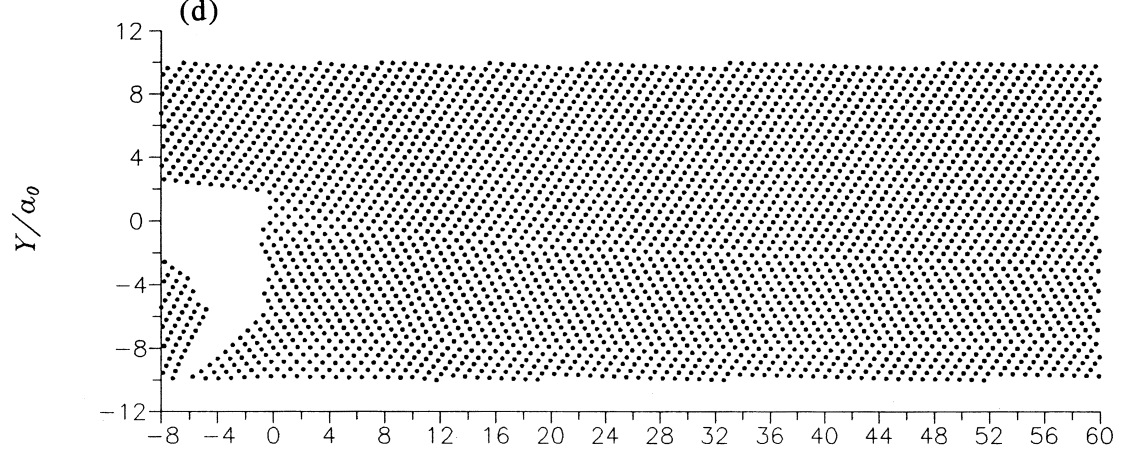

$X / a_{0}$

Fig. 8. Atomic configuration near the crack tip, deformation twinning band and crack extension under the mixed mode loads for sample 2 : (a) $K_{\mathrm{II}}=0.975 \mathrm{MPa} \mathrm{m}^{1 / 2}, K_{\mathrm{I}}=0.390 \mathrm{MPa} \mathrm{m}^{1 / 2}$; (b) $K_{\mathrm{II}}=1.240 \mathrm{MPa} \mathrm{m}^{1 / 2}, \quad K_{\mathrm{I}}=0.496 \mathrm{MPa} \mathrm{m}^{1 / 2} ; \quad(\mathrm{c}) K_{\mathrm{II}}=3.725 \mathrm{MPa} \mathrm{m}^{1 / 2}, \quad K_{\mathrm{I}}=1.490 \mathrm{MPa} \mathrm{m}^{1 / 2} ;$ (d) $K_{\mathrm{II}}=4.612 \mathrm{MPa} \mathrm{m}^{1 / 2}, K_{\mathrm{I}}=1.840 \mathrm{MPa} \mathrm{m}^{1 / 2}$. 
observed in sample 1 and the deformation twinning in sample 2. This means that the deformation twinning is not only related to the loading rate, but also to the crystal orientation.

3.2.1. Case 3. Sample 2 is simulated under the mixed mode loads and the mixed ratio is $K_{\mathrm{II}}: K_{\mathrm{I}}=5: 2$. The atomic configuration of the deformation twinning is shown in Fig. 8. As $K_{\mathrm{II}}=0.975 \mathrm{MPa} \mathrm{m}^{1 / 2}$ and $K_{\mathrm{I}}=0.39 \mathrm{MPa} \mathrm{m}^{1 / 2}$, the deformation twinning in Fig. 8(a) is less clear than that of Fig. 5(a) which is under pure shear loading. The shape of the deformation twin band in Figs 8(b)-(d) resembles that of Fig. 5. The width of the deformation twin band in Figs 8(b) and (c) is a little larger than the opening distance of the crack surface which is contributed by mode I loading.

By comparison of the simulated results of sample 2 under the pure shear loading and mixed mode loads, several common properties of deformation twinning can be obtained. The first is that the deformation twin bands are observed in two examples above and are of similar shape. The second is that the deformation twin bands will become wider with the opening distance of crack extension. The third is that the route of crack extension is basically familiar. The initial propagation of the crack is at the lower corner of the crack face and the crack propagates continuously. But as the crack propagates over the twin boundary, its direction is changed. At $K_{\mathrm{II}}=4.612 \mathrm{MPa} \mathrm{m}^{1 / 2}$ and $K_{\mathrm{I}}=1.84 \mathrm{MPa} \mathrm{m}^{1 / 2}$, the crack extension under mixed mode loads are observed easily.

3.2.2. Case 4. Sample 3 is simulated under mixed mode loads. The plane of (110) is the slip plane in a b.c.c. crystal. Both the crack extension and deformation twin can be observed in Fig. 9(a) at a load level of $K_{\mathrm{II}}=1.774 \mathrm{MPa} \mathrm{m}^{1 / 2}$ and $K_{\mathrm{I}}=0.709 \mathrm{MPa} \mathrm{m}^{1 / 2}$. The angle between the propagation direction of the deformation twin band and the crack plane is $35^{\circ}$, which is just the rotation angle around the $z$-axis by which sample 3 can be obtained from sample 2. So the twin boundary and twin direction of sample 3 are (112) and [111], respectively, which are the same as sample 2. The crack extension along the slipping plane of the crystal can easily be understood, because the slip plane

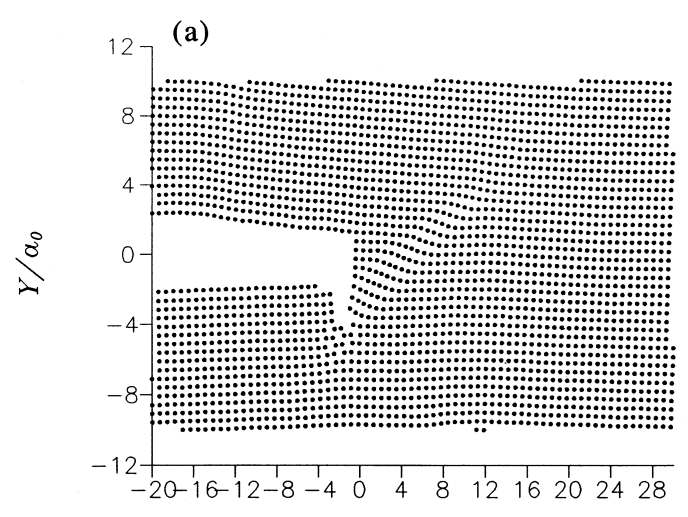

(c)

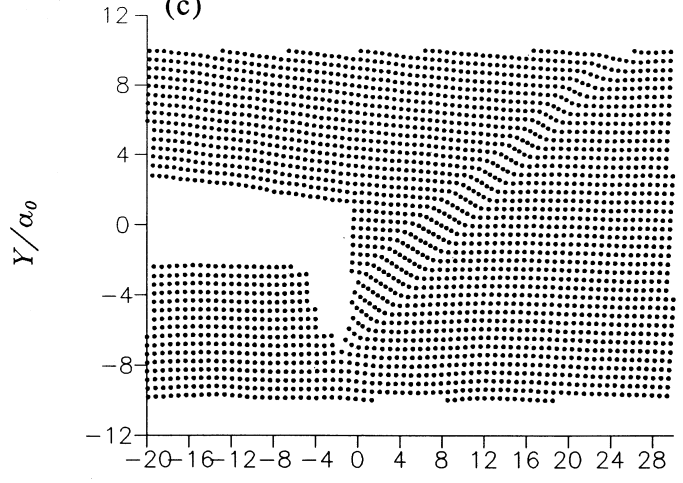

$X / a_{0}$ (b)

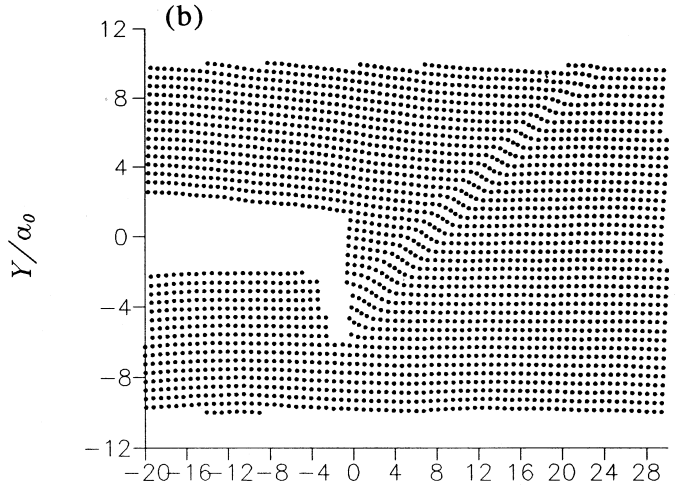

(d)

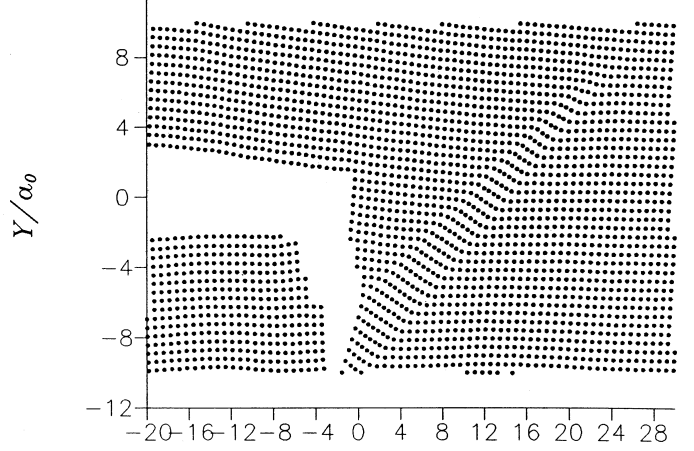

$X / \alpha_{0}$

Fig. 9. Atomic configuration near the crack tip, deformation twinning band and crack extension under the mixed mode loads for sample 3 : (a) $K_{\mathrm{II}}=1.774 \mathrm{MPa} \mathrm{m} \mathrm{M}^{1 / 2}, \quad K_{\mathrm{I}}=0.709 \mathrm{MPa} \mathrm{m}^{1 / 2}$; (b) $K_{\mathrm{II}}=2.217 \mathrm{MPa} \mathrm{m}{ }^{1 / 2}, \quad K_{\mathrm{I}}=0.887 \mathrm{MPa} \mathrm{m}^{1 / 2} ; \quad(\mathrm{c}) K_{\mathrm{II}}=2.660 \mathrm{MPa} \mathrm{m}^{1 / 2}, \quad K_{\mathrm{I}}=1.064 \mathrm{MPa} \mathrm{m}^{1 / 2} ; \quad$ (d) $K_{\mathrm{II}}=3.10 \mathrm{MPa} \mathrm{m}^{1 / 2}, K_{\mathrm{I}}=1.24 \mathrm{MPa} \mathrm{m}^{1 / 2}$. 
of a b.c.c. crystal is also the potential cleavage plane.

The simulation of sample 3 also shows that the deformation twin band propagates along the twin direction and the width of the deformation twin band is increased with the opening distance of the crack extension which is the same as the result of sample 2.

The crack propagation is along the slip plane all the time without changing its extension direction, see Figs 9(c) and (d). Steps on the crack surface can be observed which show the typical cleavage extension of the crack.

Along the $x$-direction, the stress distribution is shown in Fig. 10 at a load level of $K_{\mathrm{II}}=$ $3.10 \mathrm{MPa} \mathrm{m}^{1 / 2}$ and $K_{\mathrm{I}}=1.24 \mathrm{MPa} \mathrm{m}^{1 / 2}$. As $x / a_{0}<15$ which is in the region behind the deformation twin band, the stress distribution of the elastic solution is higher than that of MD. As $18<x / a_{0}<50$ the elastic solution is slightly higher than that of MD which is contributed by the effect of the neighboring deformation twin band. As $x / a_{0}>50$ which is in the elastic deformation state, the elastic solution is in good agreement with the simulated result.

\section{CONCLUSIONS}

Based on the fixed displacement boundary scheme of the MD simulation, this paper discusses the dislocation emission and the deformation twinning under both pure shear loading and mixed mode loads. The main results are summarized as follows:

1. The deformation twin band is observed under pure shear loading and mixed mode loads. The deformation twin band appears more clearly under pure shear loading than under mixed mode loads.

2. The simulated result shows that both the geometry orientation and stress distribution have significant effects on the nucleation and propagation of deformation twin band. The twin

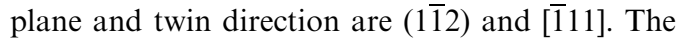
width of the deformation twin band gradually becomes narrow along the crack plane.

3. The propagating velocity of deformation twinning is about $850 \mathrm{~m} / \mathrm{s}$ which is less than half of the glide velocity of edge dislocation.

4. The effect of deformation twinning on crack extension is very important. The expansion of the deformation twin band may reduce the extension velocity of crack propagation and change the route of crack propagation.

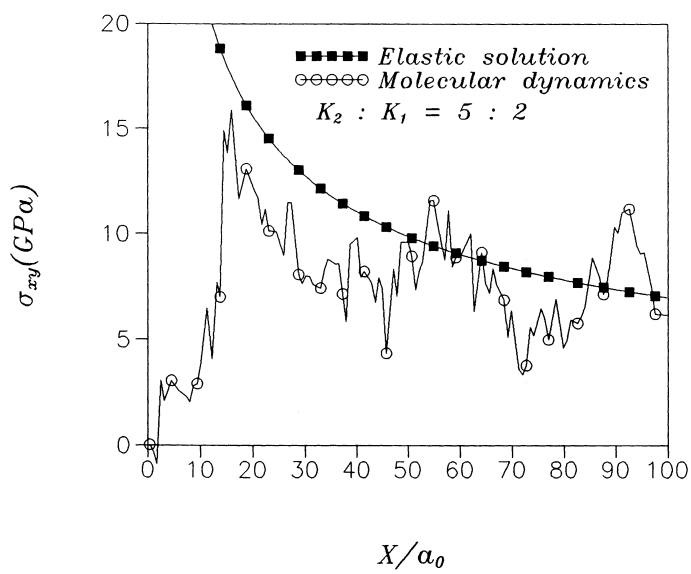

Fig. 10. Shear stress distribution along the $x$-axis under mixed mode loads for sample $3, K_{\text {II }}=3.10 \mathrm{MPa} \mathrm{m}^{1 / 2}$, $K_{\mathrm{I}}=1.24 \mathrm{MPa} \mathrm{m}{ }^{1 / 2}$

Acknowledgements - This research was supported by the National Natural Science Foundation of China (No 19704100) and the National Natural Science Foundation of Chinese Academy of Sciences (project KJ951-1-201).

\section{REFERENCES}

1. Yoo, M. H., Metall. Trans. A, 1981, 12, 409.

2. Yoo, M. H., Fu, C. L. and Lee, J. K., J. Phys. III, 1991, 1, 1065.

3. Morris, J. R., Ye, Y. Y., Ho, K. M., Chan, C. T. and Yoo, M. H., Phil. Mag. A, 1995, 72, 4138.

4. Serra, A. and Bacon, D. J., Phil. Mag. A, 1991, 63, 1001.

5. Yoo, M. H. and Lee, J. K., Phil. Mag. A, 1991, 63, 987.

6. Castaing, J., Veyssiere, P., Kubin, L. P. and Rabier, J., Phil. Mag., 1982, 44, 1407.

7. Eremenko, V. G. and Nikitenko, V. I., Physica status solidi (a), 1972, 14, 317.

8. Schlogl, S. M. and Fischer, F. D., Comput. Mater. Sci., 1996, 7, 34

9. Tang, Q. H., Wang, T. C. and Zhang, Y. W., Acta mech. sin., 1996, 12, 358.

10. Kanninen, M. F. and Gehlen, P. C., Int. J. Fract. Mech., 1971, 1, 471.

11. DeCelis, B., Argon, A. S. and Yip, S., J. appl. Phys., $1983, \mathbf{5 4}, 6864$.

12. Gumbsch, P., J. Mater. Res., 1995, 10, 2897.

13. Paskin, A., Massoumzadeh, B., Shukla, K., Sieradzki, K. and Dienes, G. J., Acta metall., 1985, 33, 1987.

14. Heermann, D. W., Computer Simulation Methods in Theoretic Physics, 2nd edn. Springer-Verlag, Berlin, 1990.

15. Woodcock, L. V., Chem. Phys. Lett., 1971, 10, 257.

16. Honeycombe, R. W. K., The Plastic Deformation of Metals, 2nd edn. Edward Arnold Ltd, London, 1968. 